\title{
Estudio de Bauhinia thonningii y el impacto de diferentes disolventes de extracción en el potencial antioxidante de la planta
}

\author{
Morales, Abdy \\ Centro de Investigaciones Psicofarmacológicas \\ Departamento de Farmacología, Fac. de Medicina, Universidad de Panamá \\ Panamá, Panamá \\ moba245@gmail.com \\ Mero, Aldahir \\ Centro de Investigaciones Psicofarmacológicas \\ Departamento de Farmacología, Fac. de Medicina, Universidad de Panamá \\ Panamá, Panamá \\ aldahirmero20@hotmail.com \\ Díaz, Maricselis \\ Centro de Investigaciones Psicofarmacológicas, Fac. de Medicina, Universidad de Panamá \\ Panamá, Panamá \\ maricselis@hotmail.com \\ Morán, Juan \\ Centro de Investigaciones Psicofarmacológicas \\ Departamento de Farmacología, Fac. de Medicina, Universidad de Panamá \\ Panamá, Panamá \\ coljamp@gmail.com \\ Samba, Nsevolo \\ Universidad Kimpa Vita, Departamento de Análisis \\ Clínicos y Salud Pública, Barrio Popular, Angola \\ Angola, África \\ israelsamba2010@hotmail.com \\ Rodilla, Jesús \\ FibEnTech- Materiais Fibrosos e Tecnologias Ambientais \\ Departamento de Química, Universidade da Beira Interior \\ Covilhã, Portugal \\ rodilla@ubi.pt
}




\section{Guerrero De León, Estela \\ Sistema Nacional de Investigación \\ Centro de Investigaciones Psicofarmacológicas \\ Departamento de Farmacología, Fac. de Medicina, Universidad de Panamá \\ Panamá, Panamá \\ ORCID: 0000-0002-0029-1827 \\ guerrerodleon@gmail.com}

\section{RESUMEN}

Bauhinia thonningii (Milne-Redhead) forma parte de la medicina tradicional de África occidental, donde sus hojas se emplean para tratar enfermedades inflamatorias e infecciosas. Investigamos el efecto antioxidante de los extractos de hojas (BAFA), corteza (BACA) y raíz (BARA) de Bauhinia thonningii, realizando extracciones con diferentes disolventes.

Valoramos la actividad antirradicalaria frente al radical DPPH, donde los extractos BARA-Acet y BARA-Eta desarrollaron una actividad antirradicalaria no inferior a Quercetina $(73,1 \pm 0,8$; $72,3 \pm 0,4$ y $77,9 \pm 4,4 \%$, respectivamente). Otros cuatro extractos, BAFA y BARA en acetona y etanol, presentaron una inhibición de más del 65\% del radical DPPH.

La actividad antirradicalaria del patrón Quercetina frente radical óxido nítrico (NO) fue de $57,7 \pm 1,2 \%$, valores superiores al $50 \%$ también fueron obtenidos con BACA-Hex, BACA-Acet y BARA-Acet.

Fueron los extractos obtenidos a partir de las hojas de Bauhinia thonningii, BAFA-Acet y BAFA-Eta, junto con BACA-Eta, los que presentaron mayor actividad frente al radical anión superóxido $\left(\bullet \mathrm{O}_{2}-\right)(50,1 \pm 3,8,51,9 \pm 2,5$ y $50,1 \pm 3,7 \%$, respectivamente), siendo similares a la eficacia demostrada por Quercetina $(53,0 \pm 0,8 \%)$. Ninguno de los extractos hexánicos desarrolló actividad antioxidante frente al radical $\bullet \mathrm{O}_{2}-$.

En los ensayos de peroxidación lipídica, todos los extractos obtenidos de las hojas y la corteza de Bauhinia thonningii presentaron un efecto inhibitorio máximo superior al $90 \%$, sin diferencias significativas cuando se compara frente al patrón Curcumina.

Concluimos que las tres partes de la planta evaluada poseen constituyentes con poder antioxidante y, además, que la mayor eficacia se obtiene cuando se emplean los disolventes etanol y acetona para la obtención de los extractos.

Palabras claves: Bauhinia tonningii, antioxidante, anión superóxido, peroxidación lipídica, óxido nítrico. 


\section{ABSTRACT}

Bauhinia thonningii (Milne-Redhead) is part of West African traditional medicine, where its leaves are used to treat inflammatory and infectious diseases. We investigated the antioxidant effect of leaf (BAFA), bark (BACA) and root (BARA) extracts from Bauhinia thonningii, performing extractions with different solvents.

We evaluated the antiradical activity against DPPH radical, where BARA-Acet and BARA-Eta extracts developed an antiradical activity not inferior to Quercetin $(73,1 \pm 0,8 ; 72,3 \pm 0,4$ y $77,9 \pm 4,4 \%$, respectively). Four other extracts, BAFA and BARA obtained with acetone and ethanol, showed more than $65 \%$ inhibition of the DPPH radical.

The antiradical activity of the Quercetin against nitric oxide radical (NO) was $57,7 \pm 1,2 \%$, meanwhile values higher than $50 \%$ were also obtained with BACA-Hex, BACA-Acet and BARA-Acet.

The extracts BAFA-Acet, BAFA-Eta, and BACA-Eta showed the highest activity against radical $\bullet \mathrm{O}_{2-}(50,1 \pm 3,8,51,9 \pm 2,5$ y $50,1 \pm 3,7 \%$, respectively), being similar to the efficacy demonstrated by Quercetin $(53,05 \pm 0,80 \%)$. Hexane extracts did not show antioxidant activity against $\bullet \mathrm{O}_{2}$ - radical.

In lipid peroxidation assay, all extracts obtained from the leaves and bark of Bauhinia thonningii exhibited a maximum inhibitory effect higher than $90 \%$, with no significant differences compared against the standard Curcumin.

We conclude that the three parts of the plant evaluated have constituents with antioxidant capacity and the highest efficacy is obtained when ethanol and acetone are used as solvents to obtain the extracts.

Keywords: Bauhinia thonningii, antioxidant, superoxide anion, lipid peroxidation, nitric oxide.

\section{INTRODUCCIÓN}

Las enfermedades crónicas y las infecciosas representan el reto de la salud pública en todos los países del mundo, a pesar del desarrollo de fármacos para el tratamiento y manejo de enfermedades como la hipertensión, la diabetes, el cáncer o el VIH/SIDA. Se ha convertido en un reto para los investigadores encontrar candidatos a fármacos útiles para el tratamiento de estas enfermedades, libres de efectos secundarios de relevancia clínica. Se requiere desarrollar estrategias innovadoras en el descubrimiento de fármacos que se aparten de las estrategias actuales de la industria farmacéutica de los "blockbusters" [1]. 
En la actualidad, un enfoque viable sería volver al origen de los fármacos y buscar en la "naturaleza" respuestas a estas necesidades, reconociendo el papel que históricamente ha desempeñado en el descubrimiento de fármacos. Así, debemos afrontar los retos que impone la salud pública mundial, apostando porque la investigación y el desarrollo de productos naturales pueda desempeñar un papel fundamental en el descubrimiento de fármacos innovadores.

En las últimas décadas se ha producido un aumento del uso de plantas medicinales para la promoción de la salud y el tratamiento de enfermedades en muchos países, incluidos los desarrollados [2]. Las plantas siguen siendo un recurso apreciado en los países de bajos ingresos. Un ejemplo es el continente africano, donde más del $80 \%$ de las poblaciones utilizan plantas para tratar diferentes problemas de salud. Bauhinia thonningii (Milne-Redhead), una planta procedente del África Occidental, se utiliza con diversos fines medicinales [3]. Se ha reportado que el extracto crudo posee actividades antilipidémicas [4], antibacterianas [5] y antiinflamatorias [6], entre otras.

Estudios fitoquímicos han revelado la presencia de compuestos químicos diversos que posiblemente sean los responsables de las actividades descritas para esta planta medicinal [7]. Será preciso valorar las propiedades de las distintas partes de esta planta, así como emplear una bateria de distintos disolventes de polaridad diferenciada $y$, a través de estudios bioguiados, llegar a concluir sobre los constituyentes con mayor potencial para convertirse en nuevos fármacos.

\section{MÉTODO}

\section{A. Actividad captadora del radical DPPH}

La determinación del porcentaje de inhibición al radical DPPH se llevó a cabo de acuerdo con la metodología descrita por Pombal et al., 2017 [8]. Empleamos microplacas de 96 pocillos, y colocamos $100 \mu \mathrm{l}$ de DPPH y $100 \mu \mathrm{l}$ del extracto a evaluar o quercetina a diferentes concentraciones $(0.24$ a $125 \mathrm{mg} / \mathrm{ml})$. Incubamos en la oscuridad durante 30 minutos y posteriormente, los datos de densidad óptica (DO) se obtuvieron a $492 \mathrm{~nm}$. Cada evaluación se realizó por triplicado. Para calcular el porcentaje de inhibición empleamos la Fórmula 1.

Fórmula 1 óde InhibiciónDPPH $=\frac{\left[D O_{D P P H}-D O_{\text {extracto }}\right]}{D O_{D P P H}} \times 10$ 


\section{B. Evaluación de la capacidad atrapadora del óxido nítrico}

Colocamos $50 \mu \mathrm{l}$ del extracto a evaluar disuelto en DMSO, según concentración a evaluar, y añadimos $50 \mu \mathrm{l}$ de una solución Nitroprusiato de Sodio (10mM) e incubamos a 25 o $\mathrm{C}$ durante 5 minutos. Adicionamos $50 \mu \mathrm{l}$ del Reactivo de Griess antes de realizar las determinaciones del radical óxido nítrico (NO) a $560 \mathrm{~nm}$ [9]. Se empleo quercetina disuelta en DMSO como control positivo.

\section{Capacidad atrapadora del anión superóxido en un sistema no enzimático}

La actividad inhibitoria frente al anión superóxido $(\bullet \mathrm{O} 2-)$ fue evaluada mediante sistema no enzimático [10]. Colocamos $50 \mu \mathrm{l}$ de los extractos a diferentes concentraciones y añadimos $50 \mu$ de cada uno de los siguientes reactivos: PMS (120 $\mu \mathrm{M})$, NADH (936 $\mu \mathrm{M})$ y NBT $(300 \mu \mathrm{M})$. La placa era incubada a $25^{\circ} \mathrm{C}$ durante 5 minutos, para posteriormente realizar las determinaciones a $560 \mathrm{~nm}$.

\section{Ensayo de inhibición de la peroxidación lipídica}

Para cada $100 \mu \mathrm{l}$ del homogeneizado de huevo (1:25, v/v en solución PBS, pH 7.4), añadimos $10 \mu \mathrm{L}$ de extracto y $50 \mu \mathrm{L}$ FeSO 4 ( $25 \mathrm{mmol} / \mathrm{l}$ ) y PBS c.s.p. $300 \mu$ l. Incubamos a 37 ${ }^{\circ} \mathrm{C}$ durante 15 minutos, y añadimos $50 \mu \mathrm{l}$ de ácido tricloroacético al $15 \% \mathrm{p} / \mathrm{v}$. Centrifugamos la muestra (3,500 rpm x 15 minutos), extraemos $200 \mu \mathrm{l}$ del sobrenadante y añadimos $100 \mu \mathrm{l}$ de ácido tiobarbitúrico. Calentamos la mezcla a $95{ }^{\circ} \mathrm{C}$ durante 30 minutos. Dejamos enfriar antes de medira la absorbancia de las muestras a $532 \mathrm{~nm}$ [11].

\section{RESULTADOS}

\section{A. Actividad captadora del radical DPPH}

El efecto antirradicalario máximo frentea DPPH, expresado como porcentaje deinhibición, obtenido con el patrón Quercetina fue de 77,9 4,3 y una actividad similar fue alcanzada por los extractos en acetona y etanol obtenido de las raíces de Bauhinia thonningii $(73,0 \pm 0,7$ y $72,2 \pm 0,4$, respectivamente). Para el resto de los extractos, los resultados obtenidos se muestran en la Tabla 1. 
Tabla 1. Valores de eficacia inhibitoria máxima (Emax) y concentración inhibitoria $50(\mathrm{Cl} 50)$ desarrollada por los extractos de hojas (BAFA), corteza (BACA) y raíz (BARA) de Bauhinia tonningii frente a los radicales DPPH y óxido nítrico. ( $p<0.05$ vs Quercetina)

\begin{tabular}{|c|c|c|c|c|}
\hline \multirow{2}{*}{ Extracto } & \multicolumn{2}{|c|}{ DPPH } & \multicolumn{2}{c|}{ Óxido Nítrico } \\
\cline { 2 - 5 } & $\begin{array}{c}\text { Emax } \\
\text { (\% de inhibición) }\end{array}$ & $\begin{array}{c}\text { Cl50 } \\
(\mathbf{m g} / \mathrm{ml})\end{array}$ & $\begin{array}{c}\text { Emax } \\
\text { (\% de inhibición) }\end{array}$ & $\begin{array}{c}\text { CI50 } \\
\text { (mg/ml) }\end{array}$ \\
\hline Quercetina & $77,9 \pm 4,3$ & 8,5 & $57,7 \pm 1,2$ & 14,3 \\
\hline BAFA-Hex & $29,8 \pm 1,5^{*}$ & $\mathrm{nd}$ & $33,9 \pm 4,1^{*}$ & $-9,7$ \\
\hline BAFA-Acet & $69,8 \pm 0,5^{*}$ & 14,3 & $38,7 \pm 3,6^{*}$ & $\mathrm{nd}$ \\
\hline BAFA-Eta & $66,6 \pm 0,9^{*}$ & 61,4 & $41,7 \pm 0,4^{*}$ & $-35,9$ \\
\hline BACA-Hex & $11,5 \pm 1,2^{*}$ & $\mathrm{nd}$ & $57,0 \pm 1,2$ & 1,8 \\
\hline BACA-Acet & $69,5 \pm 0,8^{*}$ & 3,3 & $50,4 \pm 2,5^{*}$ & $\mathrm{Nd}$ \\
\hline BACA-Eta & $65,5 \pm 0,9^{*}$ & 3,2 & $46,5 \pm 3,3^{*}$ & $\mathrm{Nd}$ \\
\hline BARA-Hex & $20,8 \pm 2,1^{*}$ & $\mathrm{nd}$ & $38,4 \pm 1,7^{*}$ & $\mathrm{Nd}$ \\
\hline BARA-Acet & $73,0 \pm 0,7$ & 37,9 & $51,5 \pm 1,2^{*}$ & 103,0 \\
\hline BARA-Eta & $72,2 \pm 0,4$ & 17,7 & $45,9 \pm 2,5^{*}$ & $-216,0$ \\
\hline
\end{tabular}

$\mathrm{nd}=$ no determinado

\section{B. Capacidad atrapadora del óxido nítrico}

La actividad antioxidante frente al radical NO para el extracto BACA-Hex fue similar a la desarrollada por quercetina $(57,0 \pm 1,2$ y $57,7 \pm 1,2$, respectivamente), mientras que valores superiores al 50\% también fueron obtenidos con BACA-Acet y BARA-Acet (Tabla 1). A pesar del uso etnobotánico que se reporta para las hojas de Bauhinia tonningii, la actividad antirradicalaria desarrollada por los extractos BAFA frente al NO fue discreta $(<40 \%$ de inhibición).

\section{Capacidad atrapadora del anión superóxido}

La actividad inhibitoria frente al $\bullet 02$ - fue similar tanto para quercetina $(53,0 \pm 0,8 \%)$, como para los extractos en acetona y etanol obtenidos a partir de las hojas de Bauhinia tonningii (BAFA-Acet: $50,1 \pm 3,8$ y BAFA-Eta: $51,9 \pm 2,5$ ). Uno de los extractos obtenidos a partir de la raíz de la planta, BARA-Eta, también presentó actividad frente al radical - $02-(50,1 \pm 3,7 \%)$. Por su parte, ninguno de los extractos apolares desarrolló actividad antioxidante en este ensayo (Figura 1). 
Fig. 1, Capacidad antioxidante máxima desarrollada por los extractos de hojas (BAFA), corteza (BACA) y raíz (BARA) de Bauhinia tonningii frente al radical anión superóxido ( $\left({ }^{\circ} \mathrm{O}_{2}\right)$.
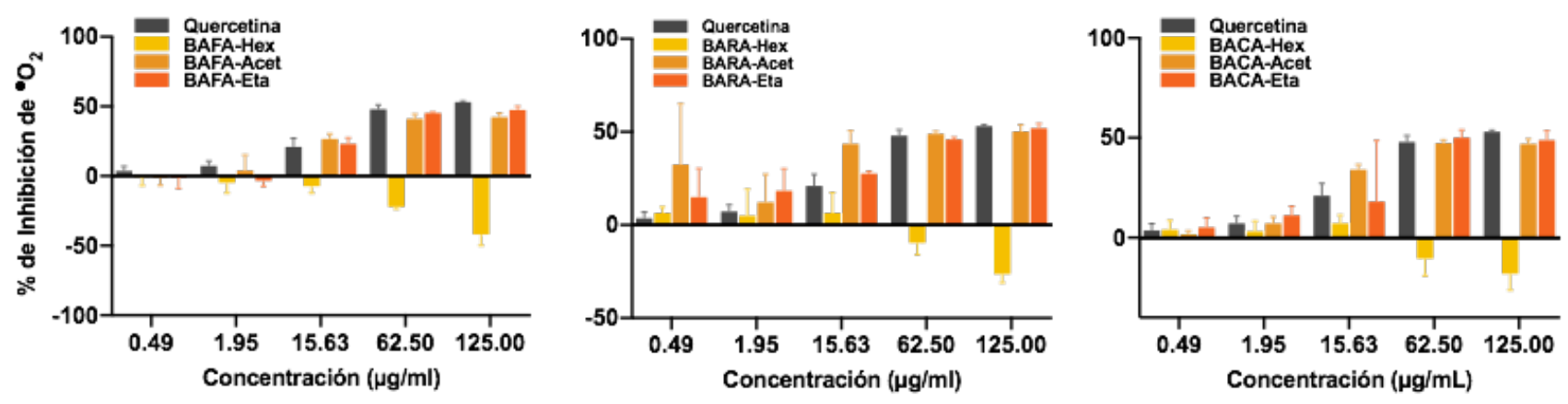

D. Inhibición de la peroxidación lipídica

Los lipoperóxidos son producto del ataque de las especies reactivas de oxígeno (ERO) a los ácidos grasos poliinsaturados que forman parte de los fosfolípidos de la membrana celular, y a los ácidos grasos presentes en los alimentos. Empleamos el extracto de yema de huevo como material de reacción para medir la capacidad que poseen los extractos sobre la formación de estos lipoperóxidos. Los resultados obtenidos reflejan una capacidad inhibitoria promedio del $90 \%$ para casi todos los extractos a la máxima concentración ensayada (Figura 2). Sólo para los extractos hexánicos de hojas y raíces de la planta, la actividad fue inferior al patrón curcumina $(71,7 \pm 0,6 ; 84,8 \pm 1,1$ y $97,2 \pm 0,3 \%$ de inhibición, respectivamente).

Fig. 2, Inhibición máxima de la peroxidación lipídica desarrollada por los extractos de hojas (BAFA), corteza (BACA) y raíz (BARA) de Bauhinia thonningii.
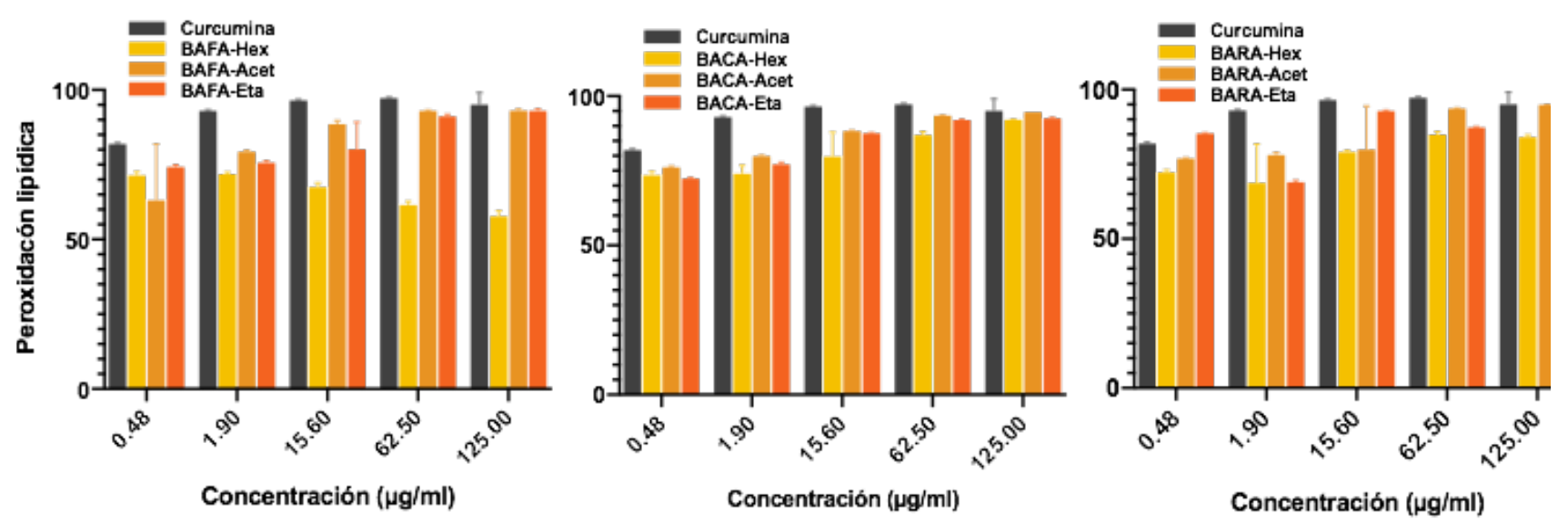


\section{CONCLUSIONES}

Empleamos diferentes radicales para medir la actividad antioxidante de un grupo de 9 extractos obtenidos a partir de Bauhinia thonningii. Observamos actividades variables según el extracto y radical al que se expone, siendo en general, los extractos en acetona y etanol los que presentan una mayor actividad frente a los radicales de importancia biológica, NO y $\bullet 22-$. Con relación a la parte estudiada, fueron los extractos de hojas y raíces quienes presentaron un mejor perfil antioxidante frente a estos radicales. Mediante el método de peroxidación lipídica demostramos que el total de los extractos poseen elevada capacidad de reducir la formación de lipoperóxidos, demostrando ser una fuente de interés para ser valorados en modelos biológicos.

\section{Agradecimientos}

Al Sistema Nacional de Investigación (Investigador Nacional/ Estela Guerrero), al financiamiento recibido por la Vicerrectoría de la Universidad de Panamá (VIP-01-07-072021-03) y la Secretaría Nacional de Ciencia, Tecnología e Innovación (PAAC-NI-2020-II-29).

\section{Referencias}

[1] N.E. Thomford, D.A. Senthebane, A. Rowe, D. Munro, P. Seele, A. Maroyi, K. Dzobo, "Natural Products for Drug Discovery in the 21st Century: Innovations for Novel Drug Discovery," Int J Mol Sci., vol. 19, no. 6, pp. 1578, June 2018.

[2] N.E. Thomford, K. Dzobo, D. Chopera, A. Wonkam, M. Skelton, D. Blackhurst, S. Chirikure, C. Dandara, "Pharmacogenomics Implications of Using Herbal Medicinal Plants on African Populations in Health Transition," Pharmaceuticals (Basel), vol. 8, no. 3, pp. 637-663, September 2015.

[3] O. Silva, S. Barbosa, A. Diniz, M. L. Valdeira, E. Gomes, "Plant Extracts Antiviral Activity against Herpes simplex Virus Type 1 and African Swine Fever Virus," International Journal of Pharmacognosy, vol. 35, no.1, pp. 12-16, January 1997.

[4] O. M. Ighodaro, J. O. Omole, "Effects of Nigerian Piliostigma thonningii Species Leaf Extract on Lipid Profile in Wistar Rats," ISRN Pharmacol. 2012;2012, 387942. September 2012.

[5] D. A. Akinpelu, E. M. Obuotor, "Antibacterial activity of Piliostigma thonningii stem bark," Fitoterapia, vol. 71, no. 4, pp. 442-443, August 2000.

[6] J. C. Ibewuike, F. O. Ogungbamila, A. O. Ogundaini, I. N. Okeke, L. Bohlin, "Antiinflammatory and antibacterial activities of C-methylflavonols from Piliostigma thonningii," Phytotherapy Research, vol. 11, no. 4, pp. 281-284, June 1997.

[7] M. Afolayan, R. Srivedavyasasri, O. T. Asekun, O. B. Familoni, A. Orishadipe, F. Zulfiqar, M. A. Ibrahim, S. A. Ross, "Phytochemical study of Piliostigma thonningii, a medicinal plant grown in Nigeria," Med Chem Res., vol. 10, pp. 2325-2330, October 2018.

[8] S. Pombal, Y. Hernández, D. Diez, E. Mondolis, A. Mero, J. Morán-Pinzón, E. I. Guerrero, J. M. Rodilla, "Antioxidant Activity of Carvone and Derivatives against Superoxide Ion," Nat Prod Commun., vol. 12, no. 5, pp. 653-655, May 2017.

[9] S. Lee, S. Sancheti, M. Bafna, S. Sancheti, S. Seo, "Acetylcholineterase inhibitory and antioxidant 
properties of Rhododendron yedoense var. Poukhanense bark," Journal of Medicinal Plants Research, vol. 5, no.2, pp. 248-254, January 2011

[10] H.-Y Lin, C.-C Chou, "Antioxidative activities of water-soluble disaccharide chitosan derivatives," Food Res Int., vol. 37, no. 9, pp. 883-889, April 2004.

[11] Y. Zhao, J. Dou, T. Wu, H. A. Aisa, "Investigating the antioxidant and acetylcholinesterase inhibition activities of Gossypium herbaceam," Molecules, vol. 8, no. 1, pp. 951-962, January 2013.

\section{Autorización y Licencia CC}

Los autores autorizan a APANAC XVIII a publicar el artículo en las actas de la conferencia en Acceso Abierto (Open Access) en diversos formatos digitales (PDF, HTML, EPUB) e integrarlos en diversas plataformas online como repositorios y bases de datos bajo la licencia CC: Attribution-NonCommercial-ShareAlike 4.0 International (CC BY-NC-SA 4.0) https://creativecommons. org/licenses/by-nc-sa/4.0/.

Ni APANAC XVIII ni los editores son responsables ni del contenido ni de las implicaciones de lo expresado en el artículo. 